\title{
EFEITO DA COMPOSIÇÃO DAS CORRENTES DO CONVERSOR DAS UNIDADES DE FCC NO DESEMPENHO CATALÍTICO DE ADITIVOS DESOx
}

\author{
Carla Maria Salerno Polato ${ }^{\#}$ José Luiz Fontes Monteiro \\ Programa de Engenharia Química, COPPE, Universidade Federal do Rio de Janeiro, CP 68502, 21941-972 Rio de Janeiro - RJ, \\ Brasil \\ Cristiane Assumpção Henriques* \\ Instituto de Química, Universidade do Estado do Rio de Janeiro, Rua São Francisco Xavier, 524, 20550-900 Rio de Janeiro - RJ, \\ Brasil
}

Recebido em 3/11/07; aceito em 22/7/08; publicado na web em 18/12/08

\begin{abstract}
INFLUENCE OF THE COMPOSITION OF THE STREAMS TO THE FCC CONVERTER UNIT ON THE CATALYTIC PERFORMANCE OF DESOx ADDITIVES. Hydrotalcite-like compounds having Mg partially replaced by $\mathrm{Cu}$ or $\mathrm{Mn}$ were prepared and used as precursors for two mixed oxides (Cu-OM50 and Mn-OM50) that were evaluated for $\mathrm{SO}_{\mathrm{x}}$ removal in the presence of $\mathrm{O}_{2}$, $\mathrm{NO}$ and $\mathrm{CO}$. Under $\mathrm{SO}_{2} / \mathrm{O}_{2}$ reaction system, $\mathrm{SO}_{x}$ removal was slightly higher over $\mathrm{Cu}-\mathrm{OM} 50$. The addition of $\mathrm{CO}$ and $\mathrm{NO}$ to the feed markedly hindered the $\mathrm{SO}_{2}$ oxidation over $\mathrm{Cu}-\mathrm{OM} 50$ while no significant effect was observed for Mn-OM50. For the regeneration step, the use of propane instead of $\mathrm{H}_{2}$ reduces regeneration capacity, mainly for Cu-OM50. Mn-OM50 was less affected by the feed composition, suggesting that it was a promising additive for $\mathrm{SO}_{\mathrm{x}}$ removal.
\end{abstract}

Keywords: $\mathrm{SO}_{\mathrm{x}}$ emission control; sulfur-transfer catalysts; $\mathrm{Mg}$,Al-mixed oxides/spinels.

\section{INTRODUÇÃO}

As recentes preocupações com as questões ambientais, que se traduzem em legislações governamentais mais rigorosas, bem como a tendência mundial de processamento de frações de petróleo cada vez mais pesadas e ricas em compostos sulfurados e nitrogenados, têm direcionado as pesquisas para a redução nas emissões de monóxido de carbono, óxidos de enxofre e óxidos de nitrogênio resultantes da queima do coque no regenerador das unidades de FCC (craqueamento catalítico fluido) existentes nas refinarias.

Uma alternativa para a redução das emissões de $\mathrm{SO}_{\mathrm{x}}$ é o uso de substâncias cataliticamente ativas em reações de oxidação como aditivos para os catalisadores de FCC. O uso de aditivos requer pouco investimento de capital, exceto pelo custo da carga de aditivo no sistema e pela disponibilidade de uma planta Claus para a recuperação do $\mathrm{H}_{2} \mathrm{~S}$ gerado no processo. A boa performance de um catalisador para remoção de $\mathrm{SO}_{x}$ encontra-se associada à sua atividade para promover os três tipos de reação que ocorrem no processo: oxidação do $\mathrm{SO}_{2}$ a $\mathrm{SO}_{3}$ nas condições de operação do regenerador (953-1003 K); quimissorção do $\mathrm{SO}_{3}$ na forma de sulfatos e, regeneração do catalisador por redução dos sulfatos sob a forma de $\mathrm{H}_{2} \mathrm{~S}$ no riser (reator de leito móvel ascendente) (793-803 K). ${ }^{1}$

No que se refere à formulação do catalisador, os óxidos básicos são considerados os aditivos mais ativos para a fixação do $\mathrm{SO}_{3}$ sob a forma de sulfato. Entretanto, os sulfatos formados com os óxidos $\mathrm{CaO}$ e $\mathrm{MgO}$ são extremamente estáveis e dificilmente se decompõem na zona de reação. A alumina também pode ser usada, mas, neste caso, devido à instabilidade térmica do sulfato de alumínio nas condições de operação do regenerador das unidades de FCC, a quantidade de $\mathrm{SO}_{3}$ fixado na alumina é relativamente baixa. Assim sendo, compostos com basicidade intermediária, tais como óxidos mistos e espinélios

\footnotetext{
*e-mail: cah@uerj.br

\#Endereço atual: Instituto Nacional da Propriedade Industrial, Pça Mauá, 7 , 22081-240 Rio de Janeiro - RJ, Brasil
}

de magnésio e alumínio, têm sido empregados de forma a encontrar um ponto ótimo entre a fixação de $\mathrm{SO}_{3}$ como sulfato e a regeneração do catalisador. ${ }^{2-5}$ Tanto os espinélios de magnésio e alumínio, estequiométricos $\left(\mathrm{MgAl}_{2} \mathrm{O}_{4}\right)$ ou não estequiométricos $\left(\mathrm{MgAl}_{2} \mathrm{O}_{4}\right.$. $n \mathrm{MgO}$ ), como os óxidos mistos dos dois metais são obtidos a partir da calcinação de precursores à base de hidroxicarbonatos de magnésio e alumínio, pertencentes a uma classe de argilas aniônicas denominadas hidrotalcitas. Como o teor de $\mathrm{SO}_{3}$ no regenerador é relativamente baixo, faz-se necessária a adição de um outro componente que possua propriedades redox. Este componente promoveria a oxidação do $\mathrm{SO}_{2}$ a $\mathrm{SO}_{3}$ no regenerador bem como a recuperação, no riser, do óxido básico pela redução das espécies sulfatadas. As referências encontradas na literatura registram a incorporação de diferentes metais $(\mathrm{Ce}, \mathrm{Cu}$, Co e Fe, por exemplo) em compostos tipo hidrotalcita (HTLC) como a maneira de gerar as propriedades oxidantes e redutoras necessárias ao seu bom desempenho na remoção do $\mathrm{SO}_{x} \cdot{ }^{2-4,6-12}$ Dentre estes metais, os catalisadores que apresentam cobre como promotor na sua composição têm se destacado dentre os vários sistemas estudados..$^{2-4,10-12}$ A introdução de Mn aparece, também, como promissora, uma vez que catalisadores contendo óxidos de manganês são utilizados com eficiência em muitos processos industriais que envolvem reações de oxi-redução, devido aos diversos estados de oxidação $(+2,+3,+4$ e $+7)$ que o elemento pode assumir. Particularmente com relação aos processos de remoção de $\mathrm{SO}_{x}$, o uso de catalisadores baseados em óxidos de manganês tem sido investigado em trapas para captura de $\mathrm{SO}_{x}$ da corrente de gases de exaustão de motores diesel ou a gasolina, com o objetivo de aumentar a vida útil dos catalisadores automotivos. ${ }^{13,14}$ Por outro lado, no que concerne aos aditivos para catalisadores de FCC, o presente trabalho tem aspecto inovador, uma vez que não se encontra na literatura uma investigação sistemática do uso de aditivos contendo manganês.

No estudo realizado, óxidos mistos de $\mathrm{Mg}$ e $\mathrm{Al}$ com razão molar $\mathrm{M}^{3+} /\left(\mathrm{M}^{2+}+\mathrm{M}^{3+}\right)$ igual a 0,50 , obtidos a partir de compostos tipo hidrotalcita contendo cobre ou manganês incorporados na sua estrutura, foram comparativamente avaliados como catalisadores para remoção 
de $\mathrm{SO}_{x}$. Na etapa de adsorção oxidativa do $\mathrm{SO}_{2}$, os testes foram realizados na presença de $\mathrm{O}_{2}$, $\mathrm{NO}$ e $\mathrm{CO}$, sob condições que visam simular aquelas existentes na fase densa dos regeneradores das unidades de FCC. Para a etapa de regeneração do catalisador, que ocorre no riser, foram empregadas comparativamente duas correntes distintas, uma contendo $30 \%$ de $\mathrm{H}_{2} / \mathrm{He}$ e outra contendo $30 \%$ de $\mathrm{C}_{3} \mathrm{H}_{8} / \mathrm{He}$.

\section{PARTE EXPERIMENTAL}

\section{Preparação dos catalisadores}

Os compostos tipo hidrotalcita (HTLCs) precursores dos óxidos mistos estudados como catalisadores foram sintetizados por coprecipitação a temperatura ambiente. Duas soluções, A e B, foram utilizadas na preparação do gel de síntese, cuja composição foi estabelecida a partir da fórmula geral $0,25 \mathrm{M}\left(\mathrm{NO}_{3}\right)_{2}: 1,25 \mathrm{Mg}\left(\mathrm{NO}_{3}\right)_{2}: 1,5$ $\mathrm{Al}\left(\mathrm{NO}_{3}\right)_{3}: 2 \mathrm{Na}_{2} \mathrm{CO}_{3}: 7,5 \mathrm{NaOH}$, sendo $\mathrm{M}=\mathrm{Cu}$ ou Mn. Uma amostra de hidrotalcita contendo apenas $\mathrm{Mg}$ e $\mathrm{Al}$ foi também preparada para servir como referência na caracterização físico-química das demais amostras sintetizadas. A solução A era preparada a partir da dissolução dos nitratos dos metais em $100 \mathrm{~mL}$ de água deionizada de modo a se obter a composição desejada para o gel de síntese $([\mathrm{Al}]+[\mathrm{Mg}]+[\mathrm{M}]=$ $1,5 \mathrm{~mol} / \mathrm{L} ;[\mathrm{Al}] /([\mathrm{Al}]+[\mathrm{Mg}]+[\mathrm{M}])=0,50 ;[\mathrm{Mg}] /[\mathrm{M}]=5)$. No preparo da solução $\mathrm{B}, \mathrm{Na}_{2} \mathrm{CO}_{3}$ e $\mathrm{NaOH}$ eram dissolvidos em água deionizada $(100 \mathrm{~mL})$ de modo a se obter uma concentração de carbonato igual a $1 \mathrm{~mol} / \mathrm{L}$ e de $\mathrm{NaOH}$ igual a $3,75 \mathrm{~mol} / \mathrm{L}$.

De acordo com o procedimento de síntese, a solução A era gradativamente adicionada à solução $\mathrm{B}$, sob agitação. $\mathrm{O}$ gel era, então, envelhecido por $18 \mathrm{~h}$, em estufa, a $333 \mathrm{~K}$ e, posteriormente, filtrado e lavado com água destilada a quente (temperatura entre 353 e 363 $\mathrm{K}$ ) até que a água de lavagem apresentasse $\mathrm{pH}=7$. O material obtido era seco em estufa, a $353 \mathrm{~K}$, por $12 \mathrm{~h}$. As amostras assim obtidas foram denominadas pela sigla M-HT50, em que M indica o metal que substitui parcialmente o $\mathrm{Mg}$ na estrutura do composto tipo hidrotalcita $(\mathrm{Cu}$ ou $\mathrm{Mn})$

Os óxidos mistos e/ou espinélios de $\mathrm{Mg}$ e $\mathrm{Al}$, contendo ainda $\mathrm{Cu}$ ou $\mathrm{Mn}$, foram obtidos por decomposição térmica dos HTLCs precursores sob fluxo de ar $(100 \mathrm{~mL} / \mathrm{min})$ da temperatura ambiente até $1023 \mathrm{~K}$, a uma taxa de aquecimento de $10 \mathrm{~K} / \mathrm{min}$, sendo, a seguir, mantidas nesta temperatura por $2 \mathrm{~h}$. Estas amostras foram denominadas pela sigla M-OM50 (OM = óxido misto), em que $\mathrm{M}$ representa $\mathrm{Cu}$ ou $\mathrm{Mn}$.

\section{Caracterização físico-química dos catalisadores}

As amostras foram caracterizadas por difração de raios X, para a identificação da(s) fase(s) presente(s) nos precursores e nos óxidos mistos/espinélios obtidos pelo tratamento térmico dos HTLCs, em um difratômetro Miniflex-Rigaku, empregando-se radiação $\mathrm{Cu} \mathrm{K} \alpha$ em uma faixa de 2 de 10 a $80^{\circ}$. A composição química foi determinada por fluorescência de raios X, em espectrômetro Rigaku, modelo Rix 3100 , controlado por computador através do software Rix 3100 e dotado de tubo gerador de raios X de Rh. A contagem dos pulsos foi feita através de um detector proporcional de fluxo.

As amostras de hidrotalcitas sintetizadas foram submetidas às análises termodiferencial (ATD) e termogravimétrica (ATG), a fim de determinar as perdas de massa associadas às etapas de desidratação e desidroxilação/descarbonatação. A análise foi realizada em termobalança Rigaku modelo PAS100, sob fluxo de ar seco (30 mL/ $\mathrm{min}$ ), numa taxa de aquecimento de $10 \mathrm{~K} / \mathrm{min}$ até $1173 \mathrm{~K}$.

A análise textural das amostras calcinadas foi realizada no equipamento ASAP (Accelerated Surface Area and Porosity) modelo 2010 da Micromeritics. Este equipamento fornece, a partir das me- didas de adsorção e dessorção do $\mathrm{N}_{2}$ a $77 \mathrm{~K}$, a área específica BET, a área e o volume de microporos pelo método t (usando a Equação de Harkins e Jura) e a área, o volume e a distribuição de mesoporos pelo método $\mathrm{BJH}$.

\section{Avaliação catalítica}

A reação foi conduzida em um micro-reator de quartzo sob pressão atmosférica. Para a etapa de adsorção oxidativa, a temperatura de reação foi fixada em $993 \mathrm{~K}$ e o tempo em $10 \mathrm{~min}$, tendo sido empregadas três correntes de composição distintas: $1630 \mathrm{ppm}$ de $\mathrm{SO}_{2}$, $1,6 \%$ (v/v) de $\mathrm{O}_{2}$ e balanço de $\mathrm{He} ; 1630$ ppm de $\mathrm{SO}_{2}, 1,6 \%$ (v/v) de $\mathrm{O}_{2}, 5 \%$ (v/v) de CO e balanço de He; 1630 ppm de $\mathrm{SO}_{2}, 1,6 \%$ (v/v) de $\mathrm{O}_{2}, 5 \%$ (v/v) de CO, 2630 ppm de $\mathrm{NO}$ e balanço de He. Para a etapa de redução do sulfato formado, foram utilizadas duas correntes com diferentes composições: $30 \%$ de $\mathrm{H}_{2}$ em He ou $30 \%$ de $\mathrm{C}_{3} \mathrm{H}_{8}$ em He. A temperatura de redução foi fixada em $803 \mathrm{~K}$, por $30 \mathrm{~min}$, e, após este tempo, a temperatura de reação era elevada até $1073 \mathrm{~K}$, numa taxa de aquecimento de $10 \mathrm{~K} / \mathrm{min}$.

Os produtos de reação foram analisados em linha através de um espectrômetro de massas quadrupolo da Balzers, modelo PrismaQMS 200, monitorando-se $\mathrm{H}_{2}(\mathrm{~m} / \mathrm{z}=2), \mathrm{H}_{2} \mathrm{O}(\mathrm{m} / \mathrm{z}=18), \mathrm{O}_{2}(\mathrm{~m} / \mathrm{z}=$ $16,32), \mathrm{H}_{2} \mathrm{~S}(\mathrm{~m} / \mathrm{z}=32,33,34), \mathrm{SO}_{2}(\mathrm{~m} / \mathrm{z}=32,48,64), \mathrm{CO}(\mathrm{m} / \mathrm{z}=$ $28), \mathrm{CO}_{2}(\mathrm{~m} / \mathrm{z}=44)$, $\mathrm{NO}(\mathrm{m} / \mathrm{z}=30)$ e $\mathrm{NO}_{2}(\mathrm{~m} / \mathrm{z}=30,46)$. As análises quantitativas foram baseadas nas áreas dos picos dos diversos componentes e seus perfis de fragmentação, com o uso de fatores de calibração e de deconvolução numérica.

\section{RESULTADOS E DISCUSSÃO}

\section{Caracterização físico-química dos catalisadores}

A Tabela 1 apresenta os resultados de caracterização química (FRX) dos compostos tipo hidrotalcita sintetizados. Os resultados mostraram-se compatíveis com a composição dos géis de síntese correspondentes.

Tabela 1. Composição química do gel de síntese e dos compostos tipo hidrotalcita sintetizados, expressa em termos de razões molares

\begin{tabular}{lcccc}
\hline \multirow{2}{*}{ Amostra } & \multicolumn{2}{c}{ Gel de Síntese } & \multicolumn{2}{c}{ Material sintetizado } \\
& $\mathrm{M} / \mathrm{Mg}$ & $\mathrm{Al} /(\mathrm{Al}+\mathrm{Mg}+\mathrm{M})$ & $\mathrm{M} / \mathrm{Mg} \mathrm{Al} /(\mathrm{Al}+\mathrm{Mg}+\mathrm{M})$ \\
\hline HT50 & 0 & 0,50 & 0 & 0,47 \\
Cu-HT50 & 0,20 & 0,50 & 0,18 & 0,46 \\
Mn-HT50 & 0,20 & 0,50 & 0,29 & 0,46 \\
\hline
\end{tabular}

$\mathrm{M}=\mathrm{Mn}$ ou $\mathrm{Cu}$

Os difratogramas de raios $\mathrm{X}$ dos compostos tipo hidrotalcita são apresentados na Figura 1. Para as três amostras observou-se a presença dos picos característicos da fase hidrotalcita na forma carbonato com estrutura lamelar e alguns picos de difração correspondentes a uma outra fase, identificada como hidróxido de alumínio, $\mathrm{Al}(\mathrm{OH})_{3}$-bayerita. ${ }^{15} \mathrm{Nos}$ compostos tipo hidrotalcita sintetizados com razão $\mathrm{Al} /(\mathrm{Al}+\mathrm{Mg})$ acima de 0,33 ocorre um aumento no número de íons $\mathrm{Al}^{3+}$ vizinhos na camada de hidróxidos. Este efeito se sobrepõe à repulsão provocada pelas cargas positivas que determinaria seu afastamento e leva à segregação da fase $\mathrm{Al}(\mathrm{OH})_{3}{ }^{16}{ }^{1} \mathrm{Não}$ foi observada segregação das fases de óxidos de cobre ou de manganês, o que sugere que estes elementos tenham sido incorporados na estrutura lamelar do HTLC. Esta proposta é reforçada pela comparação dos parâmetros de rede da fase lamelar presente nas amostras $\mathrm{Cu}-\mathrm{HT} 50$ e Mn-HT50, que foram diferentes daqueles da fase lamelar contendo apenas $\mathrm{Mg}$ e Al (amostra HT50). 


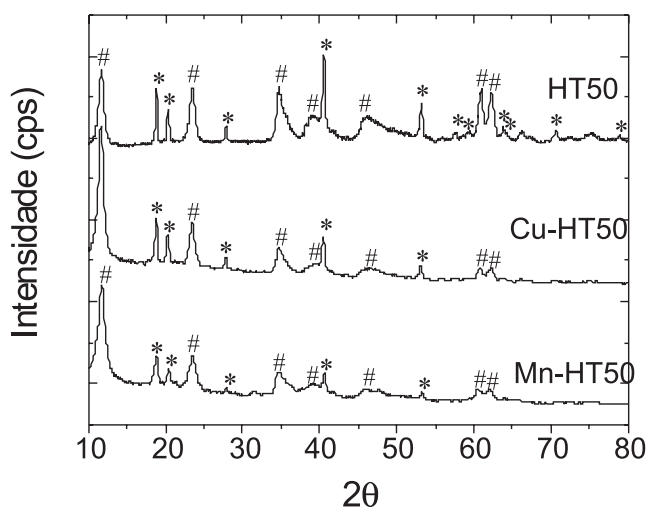

Figura 1. Difratogramas de raios $X$ das amostras sintetizadas Cu-HT50, Mn-HT50 e HT50 (referência). (\#) hidrotalcita, (*) bayerita

Os perfis de ATG e ATD correspondentes às amostras nas quais o $\mathrm{Mg}$ foi parcialmente substituído por $\mathrm{Cu}$ ou $\mathrm{Mn}$ apresentaram as duas perdas de massa típicas dos HTLCs na forma carbonato, ambas associadas a transformações endotérmicas. A primeira (400-500 K), relacionada à perda de água interlamelar, e a segunda (550-700 K), à desidratação e descarbonatação, com liberação de $\mathrm{H}_{2} \mathrm{O}$ e $\mathrm{CO}_{2} \cdot{ }^{16} \mathrm{~A}$ perda de massa observada em temperaturas intermediárias (na faixa dos $523 \mathrm{~K}$ ) pode ser associada à desidroxilação da fase bayerita presente em todas as amostras. Os resultados das análises termogravimétricas das amostras sintetizadas são apresentados na Tabela 2.

A incorporação do metal de transição influenciou os resultados de ATG/ATD, quando comparados aos do HTLC contendo apenas $\mathrm{Mg}$ e Al (HT50), tendo sido observada uma leve redução nas perdas de massa associadas às duas transformações de fase, bem como um decréscimo na temperatura na qual a taxa máxima de cada transformação foi observada, efeito este mais marcante para a mostra contendo $\mathrm{Mn}$.

Como mostrado pelos difratogramas de raios $\mathrm{X}$ apresentados na Figura 2, para a amostra HT50, o tratamento térmico a $1023 \mathrm{~K}$ determinou a segregação de uma fase óxido misto $(\mathrm{Mg}(\mathrm{Al}) \mathrm{O})$ de baixa cristalinidade com estrutura do tipo $\mathrm{MgO}$-periclásio, ${ }^{15}$ além da formação de $\gamma-\mathrm{Al}_{2} \mathrm{O}_{3}{ }^{15}$ proveniente da decomposição da bayerita. Para a amostra Cu-HT50, a calcinação sob fluxo de ar a $1023 \mathrm{~K}$ ocasionou a destruição da estrutura lamelar e levou à formação, como no caso da amostra HT50, de um óxido misto de baixa cristalinidade $(\mathrm{Mg}(\mathrm{Cu}, \mathrm{Al}) \mathrm{O})$ com estrutura tipo $\mathrm{MgO}$-periclásio. ${ }^{15}$ Além desta fase, observou-se também a formação de uma outra fase pouco cristalina, associada ao espinélio $\mathrm{MgAl}_{2} \mathrm{O}_{4}{ }^{15}$ No caso da amostra contendo Mn (amostra Mn-HT50), o tratamento térmico levou à formação de espinélios de $\mathrm{Mn}, \mathrm{Mg}$ e $\mathrm{Al}$ com estrutura cúbica ${ }^{15}$ e baixa cristalinidade.

A Tabela 3 apresenta os principais resultados de caracterização textural dos óxidos mistos obtidos a partir dos compostos tipo hidrotalcita. A análise das isotermas de adsorção/dessorção de $\mathrm{N}_{2}$ a $77 \mathrm{~K}$ das amostras calcinadas permitiu classificá-las como do tipo

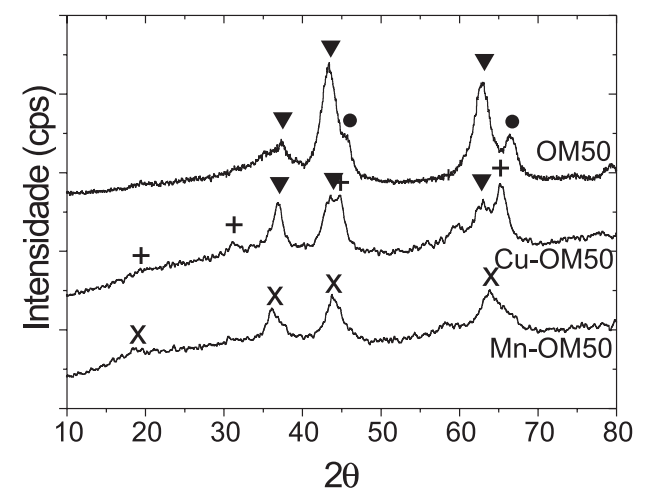

Figura 2. Difratogramas de raios $X$ dos óxidos mistos/espinélios obtidos por tratamento térmico dos precursores. (•) $\gamma_{-} \mathrm{Al}_{2} \mathrm{O}_{3}$; ( $\left.\mathbf{\nabla}\right) \mathrm{MgO}$-periclásio; (+) espinélio $\mathrm{MgAl}_{2} \mathrm{O}_{4} ;(x)$ espinélio de $\mathrm{Mn}, \mathrm{Mg}$ e Al

IV (segundo IUPAC), indicando serem as mesmas correspondentes a sólidos mesoporosos.

\section{Avaliação dos aditivos para remoção de $\mathrm{SO}_{\mathrm{x}}$}

$1{ }^{a}$ etapa: adsorção oxidativa do $\mathrm{SO}_{2}$

$\mathrm{Na}$ etapa de adsorção oxidativa de $\mathrm{SO}_{2}$ as amostras foram submetidas a três correntes de composições distintas, visando avaliar a influência da presença de $\mathrm{NO}$ e $\mathrm{CO}$ na eficiência de remoção de $\mathrm{SO}_{x}$, durante a etapa de queima do coque que ocorre no regenerador das unidades de FCC.

Para uma corrente de sulfatação composta apenas por $\mathrm{SO}_{2}$ e $\mathrm{O}_{2}$ (corrente 1), os resultados da etapa de adsorção oxidativa do $\mathrm{SO}_{x}$, mostrados nas Tabelas 4 e 5, indicam que, em relação ao óxido misto de $\mathrm{Mg}$ e $\mathrm{Al}$ (amostra OM50), a quantidade de $\mathrm{SO}_{\mathrm{x}}$ capturada pelo catalisador foi incrementada pela incorporação de $\mathrm{Mn}$ e, principalmente, de $\mathrm{Cu}$ na estrutura do HTLC precursor, sendo este comportamento catalítico relacionado às propriedades redox destes metais de transição, que atuam, assim, na promoção da oxidação do $\mathrm{SO}_{2}$ a SO . A amostra OM50 foi capaz de capturar apenas $381 \mu \mathrm{mol} / \mathrm{g}$ de $\mathrm{SO}_{2}$, enquanto as amostras Cu-OM50 e Mn-OM50 removeram, respectivamente, 3530 e $2890 \mu \mathrm{mol} / \mathrm{g}$.

Os cálculos referentes à eficiência de remoção de $\mathrm{SO}_{\mathrm{x}}$ (definida como a relação entre o número de mols de $\mathrm{SO}_{2}$ removido e o número de mols de óxido de magnésio presentes no catalisador) indicaram que aproximadamente $34 \%$ dos sítios de quimissorção presentes na amostra Mn-OM50 foram utilizados, enquanto na $\mathrm{Cu}-\mathrm{OM} 50$ este número correspondeu a $38 \%$ dos sítios. Estes valores de eficiência de remoção de $\mathrm{SO}_{\mathrm{x}}$ foram calculados admitindo-se que apenas o magnésio presente no óxido misto participa da formação de sulfato $\left(\mathrm{MgSO}_{4}\right)$, durante a etapa de adsorção oxidativa do $\mathrm{SO}_{x}$. O alumínio não foi incluído neste cálculo em função da instabilidade térmica do $\mathrm{Al}_{2}\left(\mathrm{SO}_{4}\right)_{3}$ nas condições de reação. $\mathrm{Cu}$ e $\mathrm{Mn}$ também não foram

Tabela 2. Análise termogravimétrica dos compostos tipo hidrotalcita sintetizados

\begin{tabular}{|c|c|c|c|c|c|c|}
\hline \multirow{2}{*}{ Amostra } & \multicolumn{2}{|c|}{$1^{\mathrm{a}}$ perda de massa } & \multicolumn{2}{|c|}{ perda de massa intermediária } & \multicolumn{2}{|c|}{$2^{\mathrm{a}}$ perda de massa } \\
\hline & $(\%)$ & $\operatorname{Tmax}(\mathrm{K})$ & $(\%)$ & $\operatorname{Tmax}(\mathrm{K})$ & $(\%)$ & $\operatorname{Tmax}(\mathrm{K})$ \\
\hline HT50 & 17,0 & 494 & 6,5 & 532 & 25,0 & 664 \\
\hline $\mathrm{Cu}-\mathrm{HT} 50$ & 14,2 & 469 & 7,5 & 525 & 22,4 & 646 \\
\hline Mn-HT50 & 14,8 & 458 & 6,8 & 523 & 20,8 & 620 \\
\hline $\mathrm{Al}(\mathrm{OH})_{3}^{*}$ & - & - & 34,5 & 550 & - & - \\
\hline
\end{tabular}

* bayerita 
Tabela 3. Características texturais dos óxidos mistos/espinélios

\begin{tabular}{lccc}
\hline Amostra & $\mathrm{S}_{\mathrm{BET}}\left(\mathrm{m}^{2} / \mathrm{g}\right)$ & $\mathrm{S}_{\text {Externa }}\left(\mathrm{m}^{2} / \mathrm{g}\right)^{\mathrm{a}}$ & $\mathrm{V}_{\text {meso }}\left(\mathrm{cm}^{3} / \mathrm{g}\right)^{\mathrm{b}}$ \\
\hline OM50 & 177 & 171 & 0,447 \\
Cu-OM50 & 137 & 137 & 0,458 \\
Mn-OM50 & 211 & 211 & 0,476 \\
\hline a: calculado pelo método t-plot & b: calculado pelo método BJH
\end{tabular}

Tabela 4. Efeito da composição da corrente de sulfatação sobre a quantidade de $\mathrm{SO}_{x}$ removida em 10 min de reação para a amostra $\mathrm{Cu}-\mathrm{OM} 50$

\begin{tabular}{|c|c|c|c|}
\hline Corrente de sulfatação* & 1 & 2 & 3 \\
\hline $\begin{array}{l}\text { Corrente do riser } \\
\text { (regeneração) }\end{array}$ & $30 \% \mathrm{H}_{2} / \mathrm{He}$ & $30 \% \mathrm{H}_{2} / \mathrm{He}$ & $30 \% \mathrm{H}_{2} / \mathrm{He}$ \\
\hline Consumo de $\mathrm{SO}_{2}(\mu \mathrm{mol} / \mathrm{g})$ & 3530 & 2180 & 936 \\
\hline \% regeneração & 100 & 29 & 39 \\
\hline \multirow{2}{*}{$\begin{array}{ll}\text { S liberado (\%) } & \begin{array}{c}\text { como } \mathrm{SO}_{2} \\
\text { como } \mathrm{H}_{2} \mathrm{~S}\end{array}\end{array}$} & 5 & 2 & 0 \\
\hline & 95 & 27 & 39 \\
\hline $\mathrm{X}$ global $\mathrm{SO}_{2}(\%)$ & 86 & 57 & 25 \\
\hline X global CO (\%) & - & 85 & 76 \\
\hline X global NO (\%) & - & - & 70 \\
\hline
\end{tabular}

*(1) 1630 ppm de $\mathrm{SO}_{2}, 1,6 \%$ de $\mathrm{O}_{2}$; (2) 1630 ppm de $\mathrm{SO}_{2}, 1,6 \%$ de $\mathrm{O}_{2}, 5 \%$ de $\mathrm{CO}$ ou (3) 1630 ppm de $\mathrm{SO}_{2}, 1,6 \%$ de $\mathrm{O}_{2}, 5 \%$ de $\mathrm{CO}$, 2630 ppm de NO e balanço de He.

Tabela 5. Efeito da composição da corrente de sulfatação sobre a quantidade de SOx removida em 10 min de reação para a amostra Mn-OM50

\begin{tabular}{|c|c|c|c|}
\hline Corrente de sulfatação* & 1 & 2 & 3 \\
\hline Corrente do riser & $30 \% \mathrm{H}_{2} / \mathrm{He}$ & $30 \% \mathrm{H}_{2} / \mathrm{He}$ & $30 \% \mathrm{H}_{2} / \mathrm{He}$ \\
\hline Consumo de $\mathrm{SO}_{2}(\mu \mathrm{mol} / \mathrm{g})$ & 2890 & 2674 & 2642 \\
\hline \% regeneração & 58 & 58 & 60 \\
\hline \multirow{2}{*}{ S liberado (\%) $\begin{array}{l}\text { como } \mathrm{SO}_{2} \\
{\text { como } \mathrm{H}_{2} \mathrm{~S}}^{2}\end{array}$} & 1 & 2 & 3 \\
\hline & 57 & 56 & 57 \\
\hline $\mathrm{X}$ global $\mathrm{SO}_{2}(\%)$ & 74 & 72 & 70 \\
\hline X global CO (\%) & - & 22 & 12 \\
\hline X global NO (\%) & - & - & 7 \\
\hline
\end{tabular}

*(1) 1630 ppm de $\mathrm{SO}_{2}, 1,6 \%$ de $\mathrm{O}_{2}$; (2) $1630 \mathrm{ppm}$ de $\mathrm{SO}_{2}, 1,6 \%$ de $\mathrm{O}_{2}, 5 \%$ de $\mathrm{CO}$ ou (3) 1630 ppm de $\mathrm{SO}_{2}, 1,6 \%$ de $\mathrm{O}_{2}, 5 \%$ de $\mathrm{CO}$, $2630 \mathrm{ppm}$ de $\mathrm{NO}$ e balanço de $\mathrm{He}$.

incluídos neste cálculo devido ao fato das fases $\mathrm{MnSO}_{4}$ e $\mathrm{CuSO}_{4}$ não terem sido identificadas nos estudos de difração de raios $\mathrm{X}$ das amostras sulfatadas. ${ }^{17}$

A análise comparativa dos dados referentes ao consumo de $\mathrm{SO}_{2}$ quando os catalisadores foram submetidos à corrente $1\left(\mathrm{SO}_{2}+\mathrm{O}_{2}\right)$ indicou que o $\mathrm{Cu}$ promoveu a captura de $\mathrm{SO}_{\mathrm{x}}$ de modo mais eficiente que o Mn. Entretanto, quando a composição da corrente do regenerador se tornou mais próxima da condição real, observou-se que a presença de $\mathrm{CO}$ e, principalmente, de $\mathrm{NO}$ na corrente de sulfatação alterou de modo significativo a eficiência de remoção de $\mathrm{SO}_{x}$ da amostra $\mathrm{Cu}$ OM50, que decresceu abruptamente para a corrente de sulfatação composta de $\mathrm{SO}_{2}, \mathrm{CO}, \mathrm{NO}, \mathrm{e} \mathrm{O}_{2}$, conforme mostrado na Tabela 4. Ao simular as condições da fase densa do regenerador das unidades de FCC, onde a concentração de $\mathrm{CO}$ é muito maior que a concentração de $\mathrm{O}_{2},{ }^{18}$ observou-se que, introduzindo-se apenas $\mathrm{CO}$ no sistema $\mathrm{SO}_{2}+$ $\mathrm{O}_{2}$, ocorreu uma redução no consumo de $\mathrm{SO}_{2}$ e uma elevada conversão de CO. Dada a quantidade insuficiente de oxigênio para a oxidação completa de ambos os reagentes, os resultados encontrados permitem sugerir que a oxidação do CO tenha ocorrido preferencialmente sobre o catalisador estudado. Isto porque o valor obtido experimentalmente para a conversão de CO (Tabela 4) foi superior ao previsto pela estequiometria da reação considerando-se as quantidades de $\mathrm{CO}$ e $\mathrm{O}_{2}$ presentes no meio reacional, sugerindo, assim, que parte do consumo de $\mathrm{CO}$ e do $\mathrm{SO}_{2}$ poderia estar associado à reação:

$2 \mathrm{CO}_{(\mathrm{g})}+\mathrm{SO}_{2(\mathrm{~g})} \rightarrow 2 \mathrm{CO}_{2(\mathrm{~g})}+\mathrm{S}_{(\mathrm{s})}$

como evidenciado pela presença de enxofre condensado na saída do reator, ou ainda à redução das espécies de $\mathrm{Cu}(\mathrm{II})$ existentes no catalisador. ${ }^{17}$

No caso da presença de $\mathrm{NO}$, este competiria com o $\mathrm{SO}_{2}$ pelos mesmos sítios ativos. ${ }^{18-20}$ Desta forma, poder-se-ia especular que, em presença de $\mathrm{NO}$, a reação de oxidação do $\mathrm{SO}_{2}$ tenha ocorrido em menor extensão pelo fato dos sítios ativos catalisarem preferencialmente a redução do NO, que experimentou uma conversão de $70 \%$.

$2 \mathrm{NO}_{(\mathrm{g})}+2 \mathrm{CO}_{(\mathrm{g})} \rightarrow \mathrm{N}_{2(\mathrm{~g})}+2 \mathrm{CO}_{2(\mathrm{~g})}$

Assim, óxidos mistos derivados de compostos tipo HTLCs contendo $\mathrm{Cu}$ em sua composição parecem ser aditivos eficientes para catalisar a reação entre $\mathrm{NO}_{x}$ e $\mathrm{CO}$ ou coque (redução do $\mathrm{NOx}$ pelo $\mathrm{CO}$ ou pelo coque formando $\mathrm{N}_{2}$ e $\mathrm{CO}_{2}$ ) na fase densa de regenerador, sem, contudo, serem muito ativos para a remoção simultânea de $\mathrm{SO}_{x}$. Este resultado contraria a proposta feita por Corma et al. ${ }^{4}$ ao avaliarem óxidos mistos derivados de $\mathrm{Cu}-\mathrm{HTLC}$. Segundo estes autores, as amostras contendo cobre seriam promissoras para a remoção combinada de $\mathrm{SO}_{x}$ e $\mathrm{NO}_{x}$. Entretanto, esta proposição foi feita sem que os autores ${ }^{4}$ tivessem estudado a remoção simultânea destes dois poluentes atmosféricos. Como visto no presente trabalho, a amostra $\mathrm{Cu}-\mathrm{OM} 50$ pode ser considerada o melhor aditivo para a remoção de $\mathrm{SO}_{x}$, quando avaliada na presença de uma corrente composta apenas por $\mathrm{SO}_{2} \mathrm{e} \mathrm{O}_{2}$. No caso de uma corrente com composição mais próxima daquela encontrada nas UFCC (unidades de craqueamento catalítico em leito fluido), as considerações feitas por Corma e colaboradores ${ }^{4}$ falham, pois a capacidade de remoção de $\mathrm{SO}_{x}$ da amostra contendo cobre mostrou-se extremamente influenciada pela presença de $\mathrm{CO}$ e principalmente de $\mathrm{NO}_{x}$ no meio reacional.

Wen et al. ${ }^{19,20}$ avaliaram óxidos mistos derivados de HTLCs nos quais o $\mathrm{Mg}$ foi substituído por $\mathrm{Cu}$ na remoção de $\mathrm{NO}_{\mathrm{x}}$ em presença de $\mathrm{SO}_{2}$. Observaram um decréscimo significativo da conversão de NO devido à presença do $\mathrm{SO}_{2}$ na carga, que causaria um rápido envenenamento do aditivo. Embora os autores tenham empregado condições que simulassem a fase densa dos regeneradores das unidades de FCC (corrente composta por $\mathrm{SO}_{2}, \mathrm{NO}, \mathrm{CO}$ e $\mathrm{O}_{2}$ ), as condições de reação e as técnicas empregadas para a avaliação do desempenho catalítico dos aditivos foram diferentes daquelas usadas no presente trabalho, de modo que os resultados obtidos não puderam ser diretamente comparados entre si. Wen et al. ${ }^{19,20}$ realizaram, também, estudos por espectroscopia na região do infra-vermelho dos aditivos contendo cobre. Os resultados indicaram que as espécies $\mathrm{Cu}(\mathrm{I})$ seriam os sítios ativos para a quimissorção do $\mathrm{CO}$, enquanto que as espécies $\mathrm{Cu}$ (II) seriam ativas para a adsorção do NO, mas não do CO. Segundo os autores, a competição entre $\mathrm{SO}_{2}$ e $\mathrm{NO}$ pelos sítios $\mathrm{Cu}$ (II) seria a causa da desativação observada.

Estudos termodinâmicos envolvendo as múltiplas reações associadas ao processo de remoção catalítica do SOx foram realizados 
por Pereira et al.. ${ }^{21}$ Seus resultados indicaram que a redução do $\mathrm{CuO}$ a $\mathrm{Cu}_{2} \mathrm{O}$ seria favorecida termodinamicamente mesmo em condições oxidantes. Neste caso, o $\mathrm{CuO}$ desempenharia papel ativo na etapa de oxidação do $\mathrm{SO}_{2}$, como sugerido a seguir:

$2 \mathrm{CuO}_{(\mathrm{s})}+\mathrm{SO}_{2(\mathrm{~g})} \leftrightarrow \mathrm{Cu}_{2} \mathrm{O}_{(\mathrm{s})}+\mathrm{SO}_{3(\mathrm{~g})}$

Desta forma, no caso da corrente composta por $\mathrm{SO}_{2}, \mathrm{NO}, \mathrm{CO}_{\text {e O}}$, existiria uma competição entre o $\mathrm{SO}_{2}$ e o $\mathrm{NO}$ pelas espécies $\mathrm{Cu}(\mathrm{II})$, que seriam sítios ativos tanto para a oxidação do $\mathrm{SO}_{2}$ quanto para a quimissorção do $\mathrm{NO}$ a ser reduzido pelo $\mathrm{CO}$. Nas condições estudadas neste trabalho, os resultados sugerem que a reação de redução do NO ocorre preferencialmente à de oxidação do $\mathrm{SO}_{2}$.

Para a amostra Mn-OM50, as alterações na composição da corrente de sulfatação influenciaram de modo pouco significativo a remoção de $\mathrm{SO}_{2}$, como mostrado na Tabela 5. Constata-se, assim, que este catalisador se caracteriza por ser ativo e altamente seletivo para a oxidação do $\mathrm{SO}_{2}$, sendo, porém, muito pouco ativo para catalisar reações envolvendo $\mathrm{CO}$ e $\mathrm{NO}$.

No caso do aditivo contendo manganês, o estudo termodinâmico realizado por Pereira et al. ${ }^{21}$ previu que, a $993 \mathrm{~K}$ e em presença de atmosfera oxidante, o $\mathrm{MnO}$ seria oxidado a $\mathrm{Mn}_{2} \mathrm{O}_{3}$, que, por sua vez, reagiria em grande extensão com o $\mathrm{SO}_{2}$ formando o $\mathrm{MnSO}_{4}$ :

$2 \mathrm{MnO}_{(\mathrm{s})}+1 / 2 \mathrm{O}_{2(\mathrm{~g})} \leftrightarrow \mathrm{Mn}_{2} \mathrm{O}_{3(\mathrm{~s})}$

$\mathrm{Mn}_{2} \mathrm{O}_{3(\mathrm{~s})}+2 \mathrm{SO}_{2(\mathrm{~g})}+1 / 2 \mathrm{O}_{2(\mathrm{~g})} \leftrightarrow 2 \mathrm{MnSO}_{4(\mathrm{~s})}$

Muito embora a formação de $\mathrm{MnSO}_{4}$ não tenha sido observada por difração de raios X no aditivo Mn-OM50 após a etapa de adsorção oxidativa, a presença da fase $\mathrm{MnS}$ foi identificada no difratograma de raios $\mathrm{X}$ do aditivo regenerado. ${ }^{17}$ Este fato pode ser considerado uma forte evidência da formação do $\mathrm{MnSO}_{4}$, possivelmente sob a forma de partículas muito pequenas, não detectáveis por DRX, tendo em vista ele formar $\mathrm{MnS}$ por redução em presença de $\mathrm{H}_{2}$.

A coexistência das espécies Mn(III) e Mn(II) na amostra MnOM50 foi também sugerida por análises por espectroscopia de reflectância difusa no UV-VIS. ${ }^{17}$ A comparação dos espectros da amostra antes e após a etapa de adsorção oxidativa mostrou um decréscimo na intensidade da banda associada ao $\mathrm{Mn}$ (III) com a sulfatação da amostra, o que estaria relacionado à formação do $\mathrm{MnSO}_{4}$. A formação de $\mathrm{MnS}$ (indicada pela análise por difração de raios X), por redução do $\mathrm{MnSO}_{4}$, explicaria a menor intensidade da banda correspondente ao $\mathrm{Mn}$ (III) no espectro de reflectância difusa da amostra regenerada quando comprado ao espectro da amostra calcinada.

Deste modo, tendo o manganês um papel ativo na captura do sulfato (não apenas como promotor de oxidação, mas também como sítio de fixação do $\mathrm{SO}_{3}$ ), restariam poucos sítios disponíveis para promover a reação entre o $\mathrm{CO}$ e o $\mathrm{NO}$.

\section{$2^{a}$ etapa: regeneração do catalisador}

A regeneração dos catalisadores sulfatados a partir da corrente de sulfatação $1\left(\mathrm{SO}_{2}+\mathrm{O}_{2}\right)$ foi estudada, buscando-se avaliar, também, o efeito da composição da corrente de regeneração $\left(30 \% \mathrm{H}_{2} / \mathrm{He}\right.$ ou $30 \% \mathrm{C}_{3} \mathrm{H}_{8} / \mathrm{He}$ ).

Independentemente da composição da corrente de regeneração, quando foi empregada temperatura similar àquela de operação do riser das unidades de FCC ( $803 \mathrm{~K}$ ) não foi observada a regeneração dos catalisadores sulfatados. Em função disto, foram realizados testes de TPR/MS, com aquecimento contínuo até $1073 \mathrm{~K}$, cujos resultados são apresentados nas Figuras 3 e 4, para a amostra Cu-OM50, e 5 e 6, para a amostra Mn-OM50. As análises quantitativas são apresentadas na Tabela 6 .

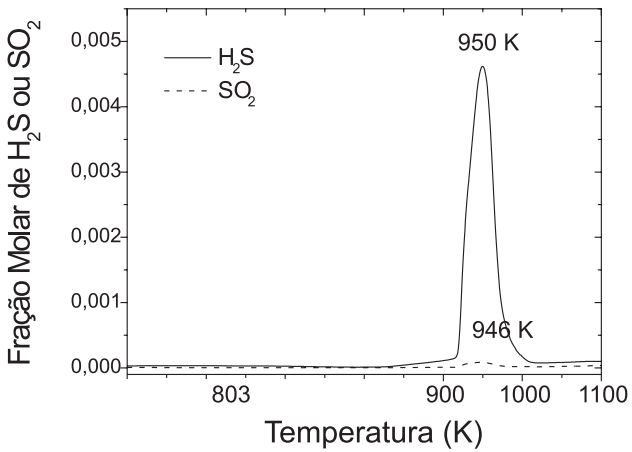

Figura 3. Perfil de redução da amostra Cu-OM50. 30\% de $\mathrm{H}_{2}$ em $\mathrm{He}$; taxa de aquecimento de $10 \mathrm{~K} / \mathrm{min}$

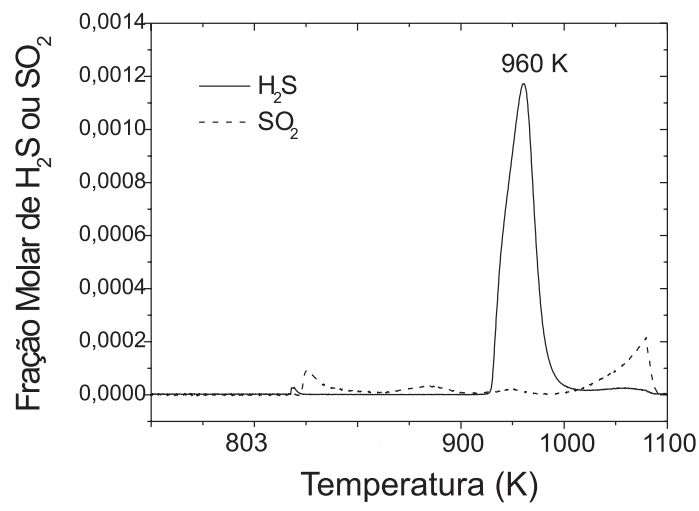

Figura 4. Perfil de redução da amostra Cu-OM50. $30 \%$ de $\mathrm{C}_{3} \mathrm{H}_{8}$ em He; taxa de aquecimento de $10 \mathrm{~K} / \mathrm{min}$

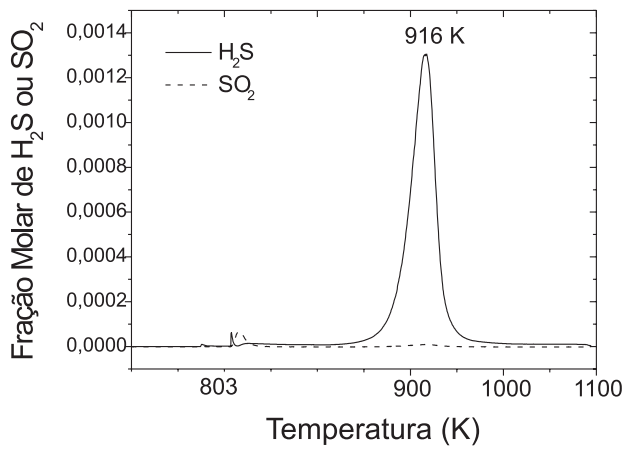

Figura 5. Perfil de redução da amostra Mn-OM50. 30\% de $\mathrm{H}_{2}$ em He; taxa de aquecimento de $10 \mathrm{~K} / \mathrm{min}$

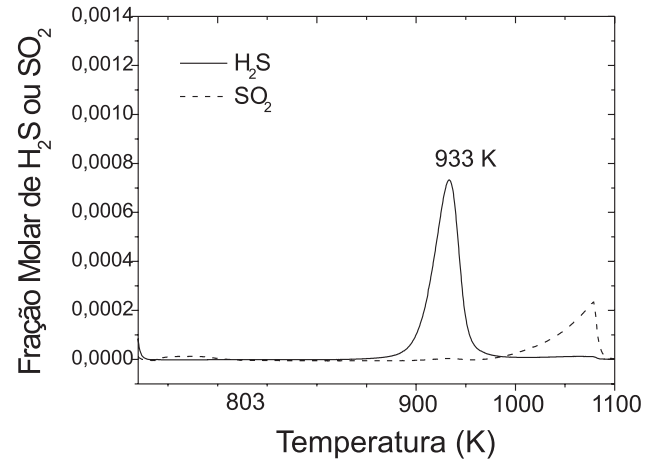

Figura 6. Perfil de redução da amostra Mn-OM50. 30\% de $\mathrm{C}_{3} \mathrm{H}_{8}$ em He; taxa de aquecimento de $10 \mathrm{~K} / \mathrm{min}$ 
Tabela 6. Efeito da composição da corrente do riser na regeneração do aditivo de remoção de SOx para uma composição da corrente de sulfatação fixa e igual a 1630 ppm de $\mathrm{SO}_{2}, 1,6 \%$ de $\mathrm{O}_{2}$

\begin{tabular}{lccccc}
\hline Amostra & \multicolumn{2}{c}{ Cu-OM50 } & \multicolumn{2}{c}{ Mn-OM50 } \\
\hline Corrente do riser* & 1 & 2 & 1 & 2 \\
Consumo de $\mathrm{SO}_{2}(\mu \mathrm{mol} / \mathrm{g})$ & 3530 & 3275 & 2890 & 2757 \\
\% regeneração & & 100 & 57 & 58 & 41 \\
& $\mathrm{SO}_{2}$ & 5 & 14 & 1 & 12 \\
S liberado (\%) & $\mathrm{H}_{2} \mathrm{~S}$ & 95 & 43 & 57 & 29 \\
\hline
\end{tabular}

*(1) $30 \%$ de $\mathrm{H}_{2}$ em He ou (2) $30 \%$ de $\mathrm{C}_{3} \mathrm{H}_{8}$ em He.

Os resultados obtidos para a amostra $\mathrm{Cu}-\mathrm{OM} 50$ indicaram a completa regeneração da amostra em presença da corrente de $\mathrm{H}_{2} / \mathrm{He}$. $\mathrm{O} \mathrm{H}_{2} \mathrm{~S}$ foi o principal produto da redução do sulfato, sendo pequena a fração reduzida parcialmente (associada à formação de $\mathrm{SO}_{2}$ ). A avaliação do perfil de redução da amostra (Figura 3) indica que a temperatura inicial para a liberação de $\mathrm{H}_{2} \mathrm{~S}$ foi superior a $850 \mathrm{~K}$ e, portanto, maior que a temperatura de reação praticada no riser da unidade de FCC. A liberação do $\mathrm{SO}_{2}$ foi simultânea à do $\mathrm{H}_{2} \mathrm{~S}$.

A substituição do $\mathrm{H}_{2}$ pelo $\mathrm{C}_{3} \mathrm{H}_{8}$ reduziu de modo significativo a regeneração do catalisador. Além disso, a análise da Figura 4 mostra um leve deslocamento do perfil de liberação do $\mathrm{H}_{2} \mathrm{~S}$ para temperatura mais elevada ( $\mathrm{T}_{\max }$ desloca-se de 950 para $960 \mathrm{~K}$ ) e um aumento da quantidade relativa de $\mathrm{SO}_{2}$ (redução parcial do sulfato), que passou a ser liberado, em sua maior parte, a temperaturas superiores a 1000 $\mathrm{K}$. Estes resultados indicaram ser a redução do sulfato fortemente influenciada pela composição da corrente do riser.

A regeneração da amostra Mn-OM50, na presença de $\mathrm{H}_{2}$, levou à liberação simultânea de $\mathrm{SO}_{2}$ e de $\mathrm{H}_{2} \mathrm{~S}$, que, também neste caso, foi liberado a temperatura superior àquela encontrada no riser da unidade de FCC (Figura 5). No caso desta amostra, como não foi descartada a possibilidade de formação de $\mathrm{MnSO}_{4}$, este poderia ser reduzido a sulfeto nas condições da reação, contribuindo também para a liberação de $\mathrm{SO}_{2}$, de acordo com a reação apresentada a seguir:

$2 \mathrm{MnSO}_{4(\mathrm{~s})}+5 \mathrm{H}_{2(\mathrm{~g})} \rightarrow \mathrm{MnO}_{(\mathrm{s})}+\mathrm{MnS}_{(\mathrm{s})}+\mathrm{SO}_{2(\mathrm{~g})}+5 \mathrm{H}_{2} \mathrm{O}_{(\mathrm{g})}$

As análises por difração de raios $\mathrm{X}$ da amostra confirmaram a formação da fase MnS (alabandita) no catalisador após a etapa de redução, sendo possível observar, também, que a estrutura tipo espinélio $\mathrm{Mn}-\mathrm{Mg}$-Al não foi recuperada, ocorrendo alterações estruturais que levaram à formação da fase $\mathrm{MgO}$, com estrutura do tipo periclásio, e do espinélio $\mathrm{MgAl}_{2} \mathrm{O}_{4} \cdot{ }^{17}$

A Figura 6 mostra que, em presença do $\mathrm{C}_{3} \mathrm{H}_{8}$, o perfil de liberação do $\mathrm{H}_{2} \mathrm{~S}$ foi levemente deslocado para temperaturas maiores $\left(\mathrm{T}_{\max }\right.$ desloca-se de 916 para $933 \mathrm{~K}$ ). Observa-se também que o uso do $\mathrm{C}_{3} \mathrm{H}_{8}$ na corrente de regeneração aumentou a quantidade de enxofre liberado como $\mathrm{SO}_{2}$ e deslocou a faixa de temperatura na qual ocorre a liberação deste composto para valores muito maiores (> $1000 \mathrm{~K})$. Tendências semelhantes foram observadas para a amostra contendo cobre.

A substituição do $\mathrm{H}_{2}$ pelo $\mathrm{C}_{3} \mathrm{H}_{8}$ reduziu a porcentagem de regeneração da amostra Mn-OM50 de modo menos significativo (de 58 para $41 \%$ ) que o observado para a amostra Cu-OM50 (100 para 57\%), o que indicaria ser o aditivo contendo manganês em sua composição menos susceptível a alterações na composição da corrente de redução no riser das unidades de FCC. Além disso, cabe ressaltar que tanto a temperatura inicial quanto a temperatura máxima de liberação de $\mathrm{H}_{2} \mathrm{~S}$ foram menores que aquelas observadas para a amostra $\mathrm{Cu}-\mathrm{OM} 50$, independentemente da atmosfera de redução estudada.
A avaliação global dos resultados indicou que o propano é um redutor menos eficiente que o hidrogênio, dados os menores níveis de redução observados para os dois catalisadores e também a maior liberação de $\mathrm{SO}_{x}$, indesejável nesta etapa do processo. Tendências similares foram observadas para o catalisador de $\mathrm{CeO}_{2}$ impregnado em óxido misto de $\mathrm{Mg}$ e $\mathrm{Al}(\mathrm{Al} /(\mathrm{Mg}+\mathrm{Al})=0,50) .{ }^{18}$

Além disso, a diferença observada entre os catalisadores com relação aos níveis de redução alcançados concorda com a proposta de Kim e Juskelis ${ }^{6}$ de que a regeneração do catalisador seria determinada não apenas pela natureza da corrente de redução, como também pela composição do mesmo. Entretanto, os resultados obtidos contrariam o mecanismo proposto por aqueles autores, ${ }^{6}$ segundo o qual a redução do $S^{6+}$ a $S^{2-}$ ocorreria por uma seqüência de reações consecutivas, tendo o $\mathrm{SO}_{2}$ como intermediário. Isto porque, em presença de $\mathrm{C}_{3} \mathrm{H}_{8}$ como agente redutor, foi possível observar claramente a liberação da maior parte do $\mathrm{SO}_{2}$ após a formação de $\mathrm{H}_{2} \mathrm{~S}$ (Figuras 4 e 6). Além disso, quando $\mathrm{o}_{2}$ foi usado como agente redutor, os perfis de redução das amostras Cu-OM50 e Mn-OM50 (Figuras 3 e 5, respectivamente) indicam que a liberação do $\mathrm{SO}_{2}$ ocorreu simultaneamente à do $\mathrm{H}_{2} \mathrm{~S}$.

\section{CONCLUSÕES}

Óxidos mistos/espinélios derivados de compostos tipo hidrotalcita (HTLC) com uma razão molar Al/( $\left(\mathrm{M}^{2+}+\mathrm{Al}\right)$ igual a 0,50 nos quais o $\mathrm{Mg}$ foi parcialmente substituído por $\mathrm{Cu}$ ou $\mathrm{Mn}$ foram preparados e avaliados como aditivos a serem incorporados aos catalisadores com o objetivo de reduzir as emissões de SOx nas unidades de FCC. Eles se mostraram mais eficientes para captura do SOx que o óxido misto contendo apenas $\mathrm{Mg}$ e $\mathrm{Al}$, o que confirma que a presença de um componente com propriedades redox é essencial para a performance adequada de um aditivo DeSOx.

A comparação da quantidade de $\mathrm{SO}_{x}$ capturado pelos aditivos $\mathrm{Cu}-$ OM50 e Mn-OM50, no caso da corrente composta apenas por $\mathrm{SO}_{2}+\mathrm{O}_{2}$, indicou que a presença do cobre promoveu a captura de $\mathrm{SO}_{x}$ de modo mais eficiente que a presença do manganês. Entretanto, a introdução de $\mathrm{CO}$ no sistema $\mathrm{SO}_{2}+\mathrm{O}_{2}$ determinou uma redução na captura de $\mathrm{SO}_{2}$ e uma elevada conversão de $\mathrm{CO}$, no caso da amostra Cu-OM50. Tendo em vista que o nível de conversão do $\mathrm{CO}$ foi superior ao previsto pela estequiometria da reação, considerando-se as quantidades de $\mathrm{CO}$ e $\mathrm{O}_{2}$ no meio reacional, acredita-se que parte do consumo do $\mathrm{CO}$ e do $\mathrm{SO}_{2}$ tenha sido devido à reação entre ambos formando $\mathrm{CO}_{2}$ e enxofre ou ainda à redução das espécies de $\mathrm{Cu}(\mathrm{II})$ existentes no catalisador. No caso da presença de NO e CO na corrente de reação, a captura do $\mathrm{SO}_{2}$ diminuiu significativamente, enquanto a reação entre $\mathrm{CO}$ e NO atingiu nível de conversão elevado. Existiria, assim, uma competição entre $\mathrm{NO}$ e $\mathrm{SO}_{2}$ pelos mesmos sítios ativos, espécies $\mathrm{Cu}$ (II), que catalisariam preferencialmente as transformações do NO. Deste modo, óxidos mistos derivados de compostos tipo HTLCs contendo $\mathrm{Cu}, \mathrm{Mg}$ e Al em sua composição parecem ser aditivos eficientes para catalisar a reação entre $\mathrm{NO}_{x}$ e $\mathrm{CO}$ na fase densa de regenerador, sem contudo serem muito ativos para remover simultaneamente o $\mathrm{SO}_{x}$.

A amostra Mn-OM50, por outro lado, mostrou-se um catalisador ativo e altamente seletivo para a remoção do $\mathrm{SO}_{2}$ da corrente de efluentes nas unidades de FCC, já que, diferentemente do observado para a amostra $\mathrm{Cu}-\mathrm{OM} 50$, a capacidade de remoção de $\mathrm{SO}_{2}$ da amostra contendo manganês não foi afetada pela presença de $\mathrm{CO}$ e, principalmente, de NO na corrente de sulfatação. No catalisador contendo manganês, este desempenharia um papel ativo não apenas como promotor de oxidação, mas também na captura do sulfato (atuaria como sítio de fixação do $\mathrm{SO}_{3}$ ), de modo que restariam poucos sítios disponíveis para promover a reação entre $\mathrm{CO}$ e NO.

Com relação à etapa de regeneração dos catalisadores sulfatados, que foi avaliada na presença de diferentes agentes de redução $\left(\mathrm{H}_{2}\right.$ 
e $\mathrm{C}_{3} \mathrm{H}_{8}$ ), embora o aditivo contendo manganês em sua composição tenha apresentado um nível de regeneração menor, ele mostrou-se menos susceptível a alterações na corrente de redução. Além disso, deve ser considerado, ainda, que na amostra Mn-OM50 tanto a temperatura inicial quanto a temperatura máxima de liberação de $\mathrm{H}_{2} \mathrm{~S}$ foram menores que aquelas observadas para a amostra $\mathrm{Cu}-\mathrm{OM} 50$, independentemente da atmosfera de redução estudada.

$\mathrm{O}$ uso de um hidrocarboneto $\left(\mathrm{C}_{3} \mathrm{H}_{8}\right)$ como agente redutor diminuiu a regeneração dos aditivos estudados e aumentou a fração de sulfato reduzida parcialmente, aumentando, conseqüentemente, a indesejável liberação de $\mathrm{SO}_{2}$.

Conclui-se, assim, que a redução das espécies sulfatadas é influenciada tanto pela composição da corrente de redução quanto pelo metal de transição presente no catalisador.

\section{MATERIAL SUPLEMENTAR}

Está disponível em http://quimicanova.sbq.org.br, na forma de arquivo .PDF, com acesso livre. São apresentados os perfis de captura do SOx presente nas diferentes correntes de alimentação pelos aditivos avaliados.

\section{AGRADECIMENTOS}

À CAPES pela bolsa de Doutorado concedida a C. M. S. Polato e ao Programa PROCIÊNCIA da UERJ.

\section{REFERÊNCIAS}

1. Cheng, W.-C.; Kim, G.; Peters, A. W.; Zhao, X.; Rajagopalan, K.; Catal. Rev. Sci. Eng. 1998, 40, 39.

2. Palomares, A. E.; Lópes-Nieto, J. M.; Lázaro, F. J.; Lopes, A.; Corma,
A.; Appl. Catal.,B 1999, 20, 257.

3. Corma, A.; Palomares, A. E.; Rey, F.; Appl. Catal.,B 1994, 4, 29.

4. Corma, A.; Palomares, A. E.; Rey, F.; Márques, F.; J. Catal. 1997, 170, 140.

5. Wang, J. A.; Chen, L. F.; Ballesteros, R.; Montoya, A.; Domínguez, J. M.; J. Mol. Catal. A: Chem. 2003, 194, 181.

6. Kim, G.; Juskelis, M. V.; Stud. Surf. Sci. Catal. 1996, 101, 137.

7. Trovarelli, A.; Leitenburg, C.; Borao, M.; Dolcetti, G.; Catal. Today 1999, 50, 353.

8. Polato, C. M. S.; Henriques, C. A.; Alcover Neto, A.; Monteiro, J. L. F.; J. Mol. Catal. A: Chem. 2005, 241, 184.

9. Wang, J. A.; Zhu, C. L.; Li, J.; J. Mol. Catal. A: Chem. 1999, 139, 31.

10. Centi, G.; Perathoner, S.; Catal. Today 2007, 127, 219.

11. Centi, G.; Perathoner, S.; Appl. Catal., B 2007, 70, 172.

12. Polato, C. M. S.; Henriques, C. A.; Rodrigues, A. C. C.; Monteiro, J. L. F.; Catal. Today 2008, 133-135, 534.

13. Li, L.; King, D. L.; Ind. Eng. Chem. Res. 2005, 44, 168.

14. Tikhomirov, K.; Kröcher, O.; Elsener, M.; Widmer, M.; Wokaun, A.; Appl. Catal., B 2006, 67, 160.

15. ICDD PDF-2 Database (Release 1998) - International Centre for Diffraction Data (ICDD). 12 Campus Boulevard Newton Square, Pennsylvania 19073-3273 USA.

16. Cavani, F.; Trifiró, F.; Vaccari, A.; Catal. Today 1991, 11, 173.

17. Polato, C. M. S.; Tese de Doutorado, Universidade Federal do Rio de Janeiro, Brasil, 2005.

18. Wen, B.; He, M.-Y.; Schrum, E.; Li, C.; J. Mol. Catal. A: Chem. 2002, 180,187

19. Wen, B.; He, M.-Y.; Appl. Catal., B 2002, 37, 75.

20. Wen, B.; He, M.-Y.; Costello, C.; Energy Fuels 2002, 16, 1048.

21. Pereira, H. B.; Polato, C. M. S.; Rodrigues, A. C. C.; Paredes, M. L. L.; Monteiro, J. L. F.; Henriques, C. A.; Anais do $14^{\circ}$ Congresso Brasileiro de Catálise, Porto de Galinhas, Brasil, 2007, CDRom. 


\section{EFEITO DA COMPOSIÇÃO DAS CORRENTES DO CONVERSOR DAS UNIDADES DE FCC NO DESEMPENHO CATALÍ́TICO DE ADITIVOS DESOx}

\section{Carla Maria Salerno Polato ${ }^{\#}$ José Luiz Fontes Monteiro}

Programa de Engenharia Química, COPPE, Universidade Federal do Rio de Janeiro, CP 68502, 21941-972 Rio de Janeiro - RJ, Brasil

\section{Cristiane Assumpção Henriques*}

Instituto de Química, Universidade do Estado do Rio de Janeiro, Rua São Francisco Xavier, 524, 20550-900 Rio de Janeiro - RJ, Brasil

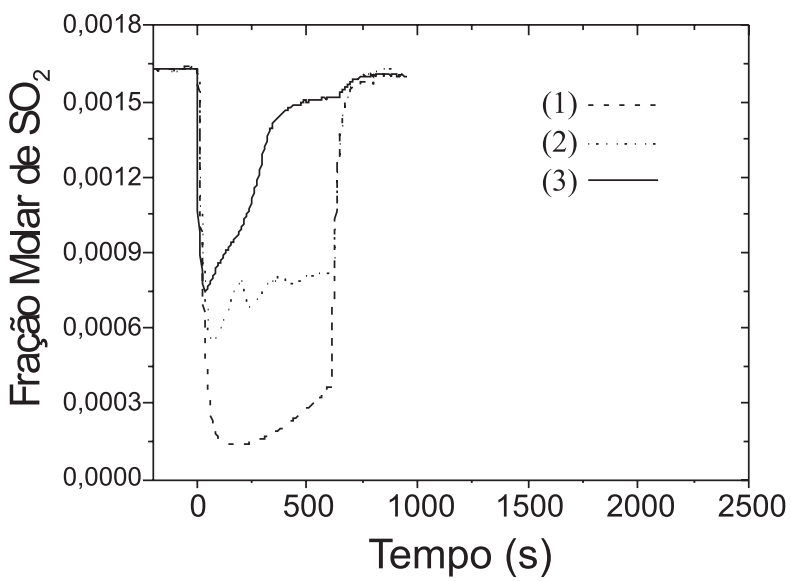

Figura 1S. Perfis de adsorção de $\mathrm{SO}_{2}$ para a amostra Cu-OM50. Condições de reação: $993 \mathrm{~K}$, (1) 1630 ppm de $\mathrm{SO}_{2}, 1,6 \%$ de $\mathrm{O}_{2}$; (2) 1630 ppm de $\mathrm{SO}_{2}, 1,6 \%$ de $\mathrm{O}_{2}, 5 \%$ de $\mathrm{CO}$ ou (3) 1630 ppm de $\mathrm{SO}_{2}, 1,6 \%$ de $\mathrm{O}_{2}, 5 \%$ de $\mathrm{CO}, 2630$ ppm de $\mathrm{NO}$ e balanço de He

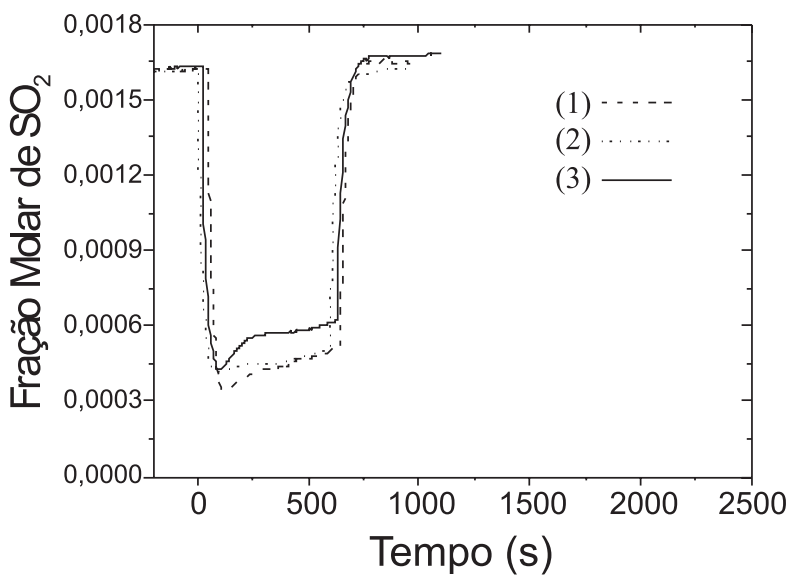

Figura 2S. Perfis de adsorção de $\mathrm{SO}_{2}$ para a amostra Mn-OM50. Condições de reação: $993 \mathrm{~K}$, (1) 1630 ppm de $\mathrm{SO}_{2}, 1,6 \%$ de $\mathrm{O}_{2}$; (2) 1630 ppm de $\mathrm{SO}_{2}, 1,6 \%$ de $\mathrm{O}_{2}, 5 \%$ de $\mathrm{CO}$ ou (3) $1630 \mathrm{ppm}^{2} \mathrm{SO}_{2}, 1,6 \%$ de $\mathrm{O}_{2}, 5 \%$ de $\mathrm{CO}, 2630$ ppm de $\mathrm{NO}$ e balanço de He 